\title{
Portafolio Digital Interactivo un Recurso para la Autoevaluación Integral
}

Interactive Digital Portfolio a Resource for Comprehensive Self-Assessment

\author{
Diego Marcelo Tipán Renjifo ${ }^{1}$ \\ https://orcid.org/0000-0002-4463-2013 \\ Universidad Tecnológica Indoamérica, Ecuador \\ Nancy de Lourdes Jordán Buenaño ${ }^{2}$ \\ https://orcid.org/0000-0002-1807-4839 \\ Universidad Tecnológica Indoamérica, Ecuador \\ Heidi Guadalupe Tipán Suárez ${ }^{3}$ \\ https://orcid.org/0000-0002-3772-7639 \\ Schlumberger, Ecuador
}

\section{Cita Recomendada}

Tipán, D., Jordán, N. y Tipán, H., (2021). Portafolio digital interactivo un recurso para la autoevaluación integral. Hamut' ay, 8 (2), 43-57, http://dx.doi.org/10.21503/hamu.v8i2.2289

\section{Resumen}

El presente estudio tiene como objetivo fomentar la cultura autoevaluativa integral en los estudiantes, mediante la implementación del portafolio digital interactivo, en los encuentros virtuales síncronos, para desarrollar su capacidad de reflexión e identificación de mejoras en el aprendizaje. La investigación se desarrolló durante la pandemia del COVID, con una población de 70 estudiantes, en los proyectos formativos de ciencias básicas, en los primeros semestres de las facultades de Arquitectura y Derecho de la Universidad Tecnológica Indoamérica; la técnica de recolección de datos fue la encuesta y el instrumento un cuestionario. La investigación tuvo un enfoque cuantitativo-cualitativo. Se concluyó que la estrategia metodológica del portafolio digital interactivo permitió fomentar la cultura de la autoevaluación integral, en el proceso de enseñanza aprendizaje, lo que hace posible que los estudiantes identifiquen sus aprendizajes y reflexionen sobre ellos, en relación con la mejora integral de los procesos educativos; experiencia que puede proyectarse más allá del encuentro síncrono, para que la Institución inclusive, robustezca su autoevaluación y acreditación.

Palabras Clave: Autoevaluación, evaluación, aprendizaje en línea, enseñanza, portafolio digital.

1. Docente Investigador; Facultad de Arquitectura-Quito-Ecuador; Maestría; diegotipan@indoamerica.edu.ec, auditortic@gmail.com. 2. Docente Investigador; Facultad de Arquitectura-Quito-Ecuador; Maestría; nancyjordan@indoamerica.edu.ec, nancyjordanb@ gmail.com.

3. Field Engineer Trainee D\&I; Schlumberger; Ingeniería; HTipan@slb.com, heiditisu@hotmail.com. 


\begin{abstract}
The present study aims to promote a comprehensive self-assessment culture in students, through the implementation of the interactive digital portfolio, in synchronous virtual encounters, to develop their capacity for reflection and identification of improvements in learning. the research was carried out during the COVID pandemic, with a population of 70 students, in the training projects of basic sciences, in the first semesters of the Faculties of Architecture and Law of the Indoamérica Technological University; the data collection technique was the survey and the instrument a questionnaire. The research had a quantitative-qualitative approach. It was concluded that the methodological strategy of the interactive digital portfolio allowed to promote the culture of integral self-evaluation, in the teaching-learning process, which makes it possible for students to identify their learning and reflect on them, regarding to the integral improvement of the educational processes; an experience that can be projected beyond the synchronous meeting, so that the Institution, strengthens its self-evaluation and accreditation as well.
\end{abstract}

Key words: Self-assessment, assessment, online learning, teaching, digital portfolio.

\section{Introducción}

Kambourova (2018), manifiesta que, durante el ejercicio docente, es importante identificar que en la planificación se deben incluir procesos evaluativos y especialmente autoevaluativos; componente que ha sido descuidado y no se ha abordado de forma holística. En el contexto virtual, los ejercicios evaluativos generalmente son pruebas que se encuentran en repositorios digitales, los cuales pueden servir para desarrollar la autoevaluación de contenidos desde bancos de preguntas; también es posible evaluar otros aspectos del proceso enseñanzaaprendizaje mediante estrategias como proyectos o resolución de problemas. Sin embargo, no se identifica en la literatura un recurso o estrategia metodológica definida para la autoevaluación durante los procesos de enseñanza-aprendizaje virtuales, en los encuentros síncronos.

Frente a lo planteado, el presente estudio tuvo el objetivo de fomentar la cultura autoevaluativa integral en los estudiantes, mediante la implementación del portafolio digital interactivo, en los encuentros virtuales síncronos, para desarrollar la reflexión e identificación de mejoras en el aprendizaje, por parte de los estudiantes.

\section{La autoevaluación}

La evaluación como categoría que abarca a la autoevaluación, se encarga de valorar los conocimientos, habilidades y valores, en el contexto de un proceso que busca formar al estudiante para que aprenda, no sólo hoy, sino también en su futuro ejercicio profesional. Para lograr esta evaluación, es necesario considerar un conjunto de insumos sobre el proceso enseñanza aprendizaje como, por ejemplo: datos, información, conocimientos, desempeños, criterios sobre el estudiante y su grupo. En este contexto, la autoevaluación se debe centrar en la reflexión crítica y en el análisis, que la persona hace sobre sí misma, en relación a un conjunto de estándares que permiten identificar debilidades y fortalezas, y con base en el estudio de estas, se deben tomar las decisiones que sean necesarias (Kambourova, 2018). Así, la autoevaluación puede integrarse dentro de un programa $y$ aportar a la mejora continua de la calidad, con la intervención de aspectos pedagógicos y tecnológicos al inicio, el desarrollo y el final del proceso formativo (Marciniak y Sallán, 2017).

La autoevaluación aplicada en conjunto con la heteroevaluación, permite mejorar la percepción estudiantil en el contexto de las competencias a desarrollarse (FernándezJiménez, Polo y Fernández, 2017). En este mismo orden y dirección, uno de los componentes 
fundamentales del proceso evaluativo es la autoevaluación; que debe ser un espacio de reflexión hacia la identificación de los logros $\mathrm{y}$ resultados que un estudiante y un grupo han alcanzado, lo que hace necesaria la recolección de evidencias que contribuyan y aseguren el logro de los aprendizajes significativos actuales, y, sobre todo, su proyección hacia el desempeño profesional.

Desde una perspectiva histórica, la autoevaluación se encuentra en los campos de la desconfianza y, los docentes no la aplican de forma planificada, estructurada y sistematizada dentro del proceso enseñanza-aprendizaje. Para abordar este fenómeno, se debe entender el concepto de lo que es la autoevaluación, desde una perspectiva integral en la que se tome al ser humano en su totalidad, desde disciplinas que permiten una reflexión más amplia sobre este término, como son la psicología, la sociología, la filosofía, la ontología, la pedagogía y la didáctica (Kambourova, 2020).

En el ámbito del aprendizaje-servicio, es posible aplicar instrumentos de autoevaluación que sirvan a las experiencias de aprendizaje de los estudiantes, que respondan a una metodología, y en los que sea importante definir el método para validar los procesos autoevaluativos (López-deArana, Aramburuzabala y Opazo, 2020); procesos que, junto a la coevaluación y heteroevaluación, generan conciencia en los estudiantes, ya que se valora el esfuerzo para alcanzar los conocimientos (Mendoza et al., 2021). Por ende, es importante incluir a la autoevaluación dentro del ámbito de acción y dominio del docente; entender que esta va más allá de lo subjetivo y afrontarla desde una perspectiva holística, en la que se reconoce, valora y evalúa la calidad humana de los estudiantes, no sólo su conocimiento; situación que lleva a considerar también un conjunto de habilidades, destrezas, afectos y emociones; lo que demanda de la educación, el logro de aprendizajes significativos, el desarrollo de actitudes hacia la práctica y el ejercicio de los principios, valores y comportamientos éticos del ser humano dentro de la sociedad.
Ante este panorama, las instituciones de educación superior deben procurar una formación integral en sus estudiantes, en la que se vincule la formación que reciben con las necesidades del mundo laboral (Díaz, 2018); así como el que combinen procesos de evaluación y autoevaluación, la formación de estudiantes críticos y reflexivos con un conjunto de habilidades de autorregulación y autoeducación, la retroalimentación del proceso educativo (Rodríguez, 2019).

Con la aplicación de instrumentos de autoevaluación, se logra la reflexión de los estudiantes en función de la resolución de preguntas que responden a criterios defactibilidad adecuados (Coria, 2017); que trascienden espacios de autoevaluación que no buscan una mejora continua sino más bien y generalmente, aspectos que permitan acreditar o certificar competencias o desempeños de estudiantes, facultades o instituciones de educación superior (Villarroel y Hernández, 2019).

\section{La Autoevaluación desde la perspectiva docente}

Al momento de contrastar los resultados de la aplicación de instrumentos de evaluación a profesores y estudiantes, se destaca su evidente diferencia, los resultados de los profesores son altos; situación que debe llevar a los profesores a desarrollar una visión autocrítica de la práctica docente en el marco de la política evaluativa de los entes evaluadores y las instituciones, para así generar mejoras en la práctica educativa, en los resultados delos estudiantes, enla sobrevaloración de la evaluación a sus profesores. Por ello, se destaca la importancia de generar una cultura autoevaluativa del profesor, una evaluación objetiva del estudiante hacia el profesor y una evaluación de la autoridad en las instituciones educativas, que se complementen y aporten una visión diferente y genere el enriquecimiento de los procesos formativos y evaluativos (Vera, Castiblanco y Cruz, 2018).

Los cambios sustanciales que se puedan dar en la docencia en función de la autoevaluación, integran una cultura evaluativa relevante para 
abordar los problemas de desempeño que se puedan presentar en el aula (Ayzum, 2017). Existen diferentes escalas e instrumentos con indicadores para la autoevaluación, que permiten identificar evidencias en relación a la mejora en la planificación, la enseñanza, la evaluación del aprendizaje y las responsabilidades profesionales de los docentes (Gutiérrez et al., 2020). Al aplicar los procesos de autoevaluación y heteroevaluación a los diferentes actores del hecho educativo, se enriquece la razón de ser de la autoevaluación; en conjunto es importante, que en los procesos evaluativos se considere la autoevaluación, pero deben existir herramientas e instrumentos que permitan generar una preparación para una educación autoevaluativa integral.

La autoevaluación integrada en el proceso de aprendizaje permite alcanzar logros, como un mayor esfuerzo y dedicación, así como una constante retroalimentación en interacción con el docente; se desarrolla la criticidad y la disciplina, además de la libertad, que brinda el poder expresarse en el aula o mediante un instrumento, a través de la autoevaluación.

Dentro de los elementos que se promueven desde la autoevaluación, se resalta el aprendizaje activo, orientado hacia el análisis y la resolución de problemas, lo que conlleva a una mejora en la manera de abordar los retos de la profesión, además, se practica el respeto yel trabajo en equipo, con el complemento de la retroalimentación del profesor, para generar en los estudiantes mayor compromiso hacia los objetivos propuestos en el curso. La autoevaluación involucra aspectos académicos, al mismo tiempo sociales y emocionales que influyen en la autocrítica del estudiante (Guzmán, 2017). La autoevaluación y la docencia necesitan estrategias definidas para mejorar el desempeño pedagógico y profesional, que lleve a prácticas reflexivas que permitan responder a la falta de criterios autoevaluativos en los docentes (Casas, 2020). Una de estas estrategias es combinarla con la coevaluación donde existe una reflexión crítica en los docentes posterior a una retroalimentación de sus pares (Alonso et. al, 2019).
La inclusión de la autoevaluación en el proceso enseñanza-aprendizaje, deja hitos importantes como el desarrollo de la autocrítica hacia el propio aprendizaje, no solo de los conocimientos que se están adquiriendo o de los logros y objetivos alcanzados; sino también del aspecto socioemocional, para generar una propuesta que lleve a mejorar la calidad de ser humano; en una educación con libertad que incorpora la autoevaluación como pilar fundamental en el proceso.

En el análisis de la autoevaluación dentro del contexto de la docencia y en la búsqueda de la mejora de sus prácticas, se destaca como un recurso de formación hacia el estudiante, que promueve el desarrollo tanto en el ámbito personal como en el ejercicio docente; proceso en el que se han de identificar las dificultades, necesidades y exigencias de la docencia actual. La autoevaluación de los docentes constituye un proceso donde las opiniones y efectividad del conocimiento que se presenta a los estudiantes, puede estimular de forma positiva las interpretaciones y valoraciones que se hacen respecto de lo que cada uno aprende, practica y crece como ser humano; la autoevaluación permite generar una valoración hacia la calidad del proceso educativo, lo que conduce a trabajar sobre intervenciones e innovaciones educativas.

La autoevaluación como instrumento que permite valorar el trabajo en aula de cada persona, en ocasiones puede relacionarse con el término balcanización, que hace referencia a la presencia de grupos en los que se genera competencia y donde cada uno de ellos procura alcanzar la supremacía en todo el conglomerado; esta comparación también puede entenderse en el contexto de un país, con una serie de ciudades independientes, que quieren obtener supremacía sobre las demás (Salom, 2018). De los anteriores planteamientos se deduce, que la autoevaluación debe ser entendida, no como una lucha por la supremacía dentro de un grupo y la auto calificación, del modo más alto posible; más bien, la autoevaluación en el contexto de la docencia debería ser una herramienta, que permita identificar necesidades y dificultades; las mismas 
que una vez identificadas permitirán que el proceso enseñanza-aprendizaje sea más integral; por lo que se debe promover la autoevaluación entre los docentes y entre los estudiantes y proponer las innovaciones educativas, que sean necesarias para lograr que haya un ejercicio introspectivo de todos los actores educativos.

Las estrategias de autoevaluación en el contexto de la innovación para el mejoramiento de las asignaturas, utiliza instrumentos de análisis como las tablas de observación sobre el desempeño didáctico docente, las evaluaciones hacia los estudiantes o las encuestas de opinión; instrumentos que miden aspectos como la buena práctica educativa, la utilización de espacios virtuales y presenciales; se convierte en una herramienta para el aprendizaje del estudiante, que integrada al ejercicio docente, posibilita redefinir la construcción de saberes donde los estudiantes en su diversidad construyan su aprendizaje, desde la diferencia de sus características y particularidades, propias de su formación secundaria. Aquí, se destaca la importancia y necesidad de contar con instrumentos de naturaleza tecnológica para el seguimiento y la organización, con el fin de mejorar la comunicación y la interacción docente-estudiante (Rico-Reintsch, 2019).

Cada docente debe guiarse por la política autoevaluativa institucional, pero sobre todo debe generar métodos autoevaluativos que sean pertinentes al contexto y en relación a las competencias que se quieran potenciar; el docente debe ser el primero en autoevaluarse, siempre en la claridad de que es un ser inacabado y de que existen múltiples aristas en las que necesita autoevaluarse, los saberes, las emociones, las actitudes, la prospectiva que tenga respecto de sí mismo y de su profesión, lo que quiere que cada uno de sus estudiantes alcance. Frente a un proceso de aprendizaje complejo y la autoevaluación como uno más de sus ingredientes, la mejora del rendimiento académico no debe ser el único objetivo a lograr durante un proceso enseñanzaaprendizaje; sumado a la construcción del saber, es importante tener conciencia sobre el ser humano en su totalidad, para ubicar objetivos reales de un proceso interno de evaluación. De no tenerse en cuenta estos aspectos, la escasa consideración a la integralidad del ser humano al momento de construir el aprendizaje, genera estudiantes que no investigan, poco reflexivos y sin instrumentos reales de autovaloración y con escasa posibilidad de diálogo consigo mismo y con sus pares.

\section{Autoevaluación en el micro currículo}

La autoevaluación debe considerarse de forma sistémica desde el macro hasta el micro currículo. Para Dooner et al., (2016), la evaluación a las instituciones se reconoce por su utilidad en los ajustes y mejoramiento requerido; así como por la posibilidad que brinda a la redefinición de aspectos relevantes dentro de la universidad. La autoevaluación presta atención a la planificación curricular y el perfil de egreso, a la relación con la actualidad, a los contenidos y metodologías de enseñanza actualizadas; aspectos que impactan todos los niveles curriculares: el aprendizaje, la gestión, la academia, el talento humano y la vinculación con la sociedad.

A lo largo de los planteamientos efectuados, se evidencia que los procesos de autoevaluación de las instituciones con fines de acreditación, han tenido resonancia, dentro de las instituciones al implementarse estructuras autoevaluativas, que responden a una visión interna de la calidad de la educación; uno de los aspectos, que son observados, es el académico, entre ellos, la gestión del currículo. Como ya se ha aclarado, la autoevaluación hacia los aprendizajes obtenidos por los estudiantes dentro de sus clases, debería tener una preponderancia alta, lo que no es el caso en los tiempos actuales. Es así que, la implementación de la autoevaluación en el nivel del micro currículo, permite operacionalizar los niveles superiores; aquí es donde se visibiliza la gestión efectuada por docentes, autoridades y el resultado en sí, del sistema educativo. Por lo que, es necesario proponer recursos, herramientas o instrumentos que posibiliten generar una conciencia autoevaluativa en los estudiantes, con el fin de promover la mejora de sus aprendizajes, 
en las que se considere al ser humano en su integralidad.

\section{Autoevaluación en la virtualidad}

La evaluación en programas de educación a distancia y en línea, debe ser vista como posibilidad de mejora de la calidad educativa. En ella, la autoevaluación ha de permitir identificar información, que luego será objeto de análisis para reconocer tanto las fortalezas como las debilidades. Lo anterior es posible con la creación de instrumentos autoevaluativos, que permitan definir ejes, categorías de indicadores y promover la cultura autoevaluativa institucional. Los elementos que están siempre presentes en la estructura de la educación a distancia, son la falta de presencialidad del proceso enseñanzaaprendizaje, la interacción con estudiantes de tipo sincrónico y asincrónico, además de recursos elaborados mediante las Tecnologías de la Información y la Comunicación (TIC); contexto en el que la autoevaluación es parte de un proceso en el que intervienen los planes de estudio, los docentes, los estudiantes, las TIC y la gestión en la administración de la institución educativa (Márquez, 2017).

Aunque se reconoce a la autoevaluación como una herramienta pedagógica válida, es importante no reproducir lo desarrollado en la presencialidad hacia la virtualidad, sin haber realizado las correspondientes consideraciones y adaptaciones de tipo didáctico, como resultados y logros; de lo contrario, es posible que se cometan errores y se realicen autoevaluaciones inadecuadas en la modalidad virtual (Amaro y Chacín, 2017). En este trabajo, es posible considera estrategias lúdicas al momento de autoevaluar a estudiantes y docentes, dado que son recibidas con mucha aceptación y motiva el desarrollo del proceso enseñanza-aprendizaje al integrarlos a los entornos virtuales (Archuby, Sanz y Pesado, 2017). En el momento en que los docentes se autoevalúan, se pueden reducir brechas alrededor del dominio de sus competencias de orden tecnológico y comunicacional, con base en un diseño instruccional definido y con la aplicación de los recursos y materiales didácticos pertinentes (Howlet, Aguilar y Diez, 2019).

Debido a que el proceso de enseñanza-aprendizaje y la autoevaluación en la presencialidad tienen sus propias estrategias y herramientas, estas no deben ser llevadas de forma textual a la virtualidad. En lo virtual con la mediación de las TIC, un proceso autoevaluativo ha de tener en cuenta los resultados y logros de aprendizaje que debe alcanzar el estudiante, los recursos y los espacios virtuales en los que se genera en el proceso enseñanza-aprendizaje y la planificación del docente.

La aplicación de las TIC, orientadas hacia la construcción del aprendizaje, redefinen las carreras universitarias al producir innovaciones en el contexto de la virtualidad e incluir recursos y herramientas educativas que van más allá de complementar la formación en línea. En lo virtual han de determinarse las limitaciones tecnológicas y evaluativas, la diversidad de herramientas disponibles, así como los canales adecuados para la evaluación y la formación integral de un profesional (Aguiar y Velásquez, 2018).

Al momento de autoevaluar es posible aplicar tanto en la presencialidad como en la virtualidad distintos instrumentos, metodologías $y$ estrategias, entre las que se encuentran la rúbrica o el estudio de caso, los cuales logran preparar al estudiante en el camino de la autonomía y la autorreflexión (Calle-Álvarez, 2020); quien tiene ciertos niveles de satisfacción respecto del desarrollo de los conocimientos propios, como herramienta que contribuye al aprendizaje autónomo y el desarrollo de la responsabilidad, que puede orientarse hacia la preparación de evaluaciones finales en el proceso enseñanza aprendizaje (Padilla, 2017).

La autoevaluación puede ser un complemento orientador para el aprendizaje en la virtualidad, ya que en este contexto existe flexibilidad en espacio y en tiempo, lo que requiere el desarrollo de procesos autoevaluativos para generar compromisos e impulsar a los estudiantes para alcanzar las metas propuestas (Sierra, 2020). En este orden de ideas, siempre existirán factores 
a favor y en contra de la implementación de la evaluación en entornos virtuales de enseñanzaaprendizaje. Por ello, se debe promover la evaluación, la autoevaluación y la coevaluación entre los docentes y los estudiantes; el empleo de estrategias metodológicas pertinentes y confiables; la implementación de recursos y estrategias evaluativas virtuales; la redefinición de enfoques pedagógicos, metodológicos y tecnológicos; con el propósito de ir más allá de asignar una nota y construir el ejercicio autoevaluativo bajo una reflexión íntegra de lo cualitativo y cuantitativo.

Desde la docencia se pueden efectuar diversas acciones en relación con la autoevaluación del aprendizaje en plataformas virtuales, como espacios en los cuales se pueden aplicar exámenes orales, teóricos y prácticos, desarrollar proyectos o realizar pruebas de carácter objetivo. En lo virtual, la autoevaluación se puede sustentar en un conjunto de reactivos o preguntas de diferente de carácter objetivo, que conforman pruebas aplicadas de forma transversal a través de la web, en la que se cuenta con gestores de preguntas y repositorios o contenedores de resultados (García et al., 2016). Sin embargo, si la autoevaluación sólo se centra en el contenido, en múltiples intentos y en señalar una respuesta correcta, no es posible configurarlas de manera integral.

Es por ello, que desde la educación se debe promover la formación de personas responsables como reflejo de la labor docente; para ello, la autoevaluación permite el desarrollo profesional y mejorar la condición de docente, al aprovechar las TIC en mejorar los desempeños. En este sentido se pueden aplicar escalas de evaluación, cuyo objetivo sea la promoción de acciones reflexivas, que fortalezcan el perfil profesional de los docentes, como parte de la búsqueda de la calidad educativa vinculada con los múltiples esfuerzos que se realizan desde las instituciones para obtener acreditaciones y certificaciones. Existen diversos modelos para la evaluación docente. En unos, el profesor es evaluado con escalas, dónde él no participó en su construcción y por otro lado los modelos se orientan hacia el logro de resultados relacionados con lo que los estudiantes puedan alcanzar y con la evidencia de cómo, el entorno contribuye de forma relevante en los resultados obtenidos. En otros modelos, la actuación docente es la premisa para evaluarlo, pero una vez más, esto depende de quién evalúa y del entorno educativo (Martínez-Izaguirre et al., 2018).

Cabe mencionar que, las evaluaciones sustentadas en instrumentos y escalas permiten identificar las fortalezas y debilidades, que persigue la mejora de la calidad educativa. Las evaluaciones basadas en la actuación docente, aunque favorecen la formación del docente, al ser tan múltiples y diversas las actividades que el docente debe desarrollar en todos los ámbitos cognitivos destrezas y valorativos, siempre será necesaria la reflexión alrededor de los logros alcanzados. Es así que la reflexión para una autoevaluación debe tener un marco y un tiempo definido, que se le debe asignar al docente para estar en condiciones de proponer mejoras, frente a las necesidades y dificultades identificadas (Carlos, Martínez y Verdejo, 2017).

\section{El portafolio - Instrumento de autoevaluación}

Una de las deficiencias de los docentes en la virtualidad es la falta de competencias para la elaboración de materiales e instrumentos didácticos, en este sentido es importante que se identifiquen las características de los estudiantes y los objetivos establecidos y cómo se van a fusionar en el contexto de las TIC (Howlet, Aguilar y Diez, 2019). Los instrumentos existentes en las plataformas de enseñanza-aprendizaje como foros, chats y demás elementos, potencian los procesos autoevaluativos y permiten el rediseño y la creación de recursos e instrumentos innovadores (Ceballo, 2018). La utilización de las TIC con fines autoevaluativos permite el manejo de imágenes virtuales con textos explicativos, que gestionadas por el mismo estudiante posibilitan el desarrollo de prácticas de contenidos específicos (García-Mauriño et al., 2017).

La autoevaluación desde su concepción formativa posibilita la retroalimentación del 
trabajo autónomo y el desarrollo de la autocrítica en los estudiantes, que al combinarse con los recursos virtuales, la indagación y la experiencia, desarrollan su autonomía y autorreflexión (Abal et al., 2020). En el proceso evaluativo, con las TIC se generan informes de gestión sobre los componentes utilizados al momento de desarrollar las clases, entre los que se destacan mensajes, foros, tareas y actas (Amorós-Poveda, 2020). Es aquí donde emerge el portafolio como escenario para la reflexión sobre los aprendizajes, desde un contexto presencial a uno virtual, como estrategia metodológica factible posible de implementar no sólo en la presencialidad, sino también en la semi-presencialidad y en el aprendizaje en línea; que al ser desarrollado, permita una autoevaluación integral.

Como lo señalan Aris y Fuentes (2016), el portafolio combinado con el desarrollo de talleres y la autoevaluación, pueden orientar el trabajo en las diferentes asignaturas, como posibilidad de reflexión y autovaloración del estudiante, en cuya elaboración es importante analizar aspectos como el esfuerzo, el grado de participación y las propuestas de mejora que puede generar. Así, el portafolio es una herramienta de recopilación útil para facilitar la identificación de lo más representativo; para superar la simple recolección de las evidencias, que se puedan almacenar; para mostrar el desarrollo del pensamiento y explicar cómo se construye el aprendizaje y la toma de decisiones; de esto se concluye que potencia la autonomía del estudiante, quien recibe de mejor manera una autoevaluación individual a una en equipo, destacándose el desarrollo de la autoconciencia.

La aplicación del portafolio ha de ir unido a la coevaluación y la autorreflexión, como parte de una propuesta docente en la que se comparten y delegan responsabilidades, para que los estudiantes tengan mayor capacidad reflexiva y crítica y tomen mejores decisiones (Márquez, 2017). Durante el diseño curricular se puede implementar la creación del portafolio virtual con componentes multidisciplinarios y transversales, éticos e interculturales. Con el portafolio se puede evaluar el aprendizaje al incorporarse al proceso de enseñanza en entornos colaborativos, bajo la planificación y evaluación de actividades en una plataforma virtual (Fosado et al., 2018); se propicia el desarrollo de destrezas y habilidades en los ámbitos conceptuales prácticos y socioemocionales (Ruano y Ballesteros, 2017); se incluyen estrategias que solucionen los problemas educativos actuales; se motiva a los estudiantes a participar en clases dinámicas e interactivas mediadas por las TIC (Montaño, 2021).

El portafolio virtual se destaca porque promueveel desarrollo del pensamiento reflexivo en contextos como la interculturalidad y contribuyen a una formación en línea integral (Sartor, 2018). Así, se constituye en una herramienta de aprendizaje y de evaluación donde las producciones de estudiantes y profesores permiten el intercambio de información y la interacción a partir de una gestión adecuada (Herrera et al., 2020). Como consecuencia de lo anterior, la aplicación del portafolio en el proceso enseñanza-aprendizaje ha de tener en cuenta la autorreflexión, la autocrítica, el desarrollo del pensamiento y mejorar la capacidad de la toma de decisiones; convirtiéndose en una herramienta o estrategia metodológica para el desarrollo de una autoevaluación integral continua que forma mejores seres humanos.

\section{El portafolio digital interactivo}

Según Tipán (2021), no se cuenta con una herramienta que permita reflexionar sobre los aprendizajes obtenidos en cada clase y la identificación de contenidos, que enfoquen la atención en las habilidades, en el aprendizaje de valores y en aspectos afectivos-emocionales, al momento de aprender 


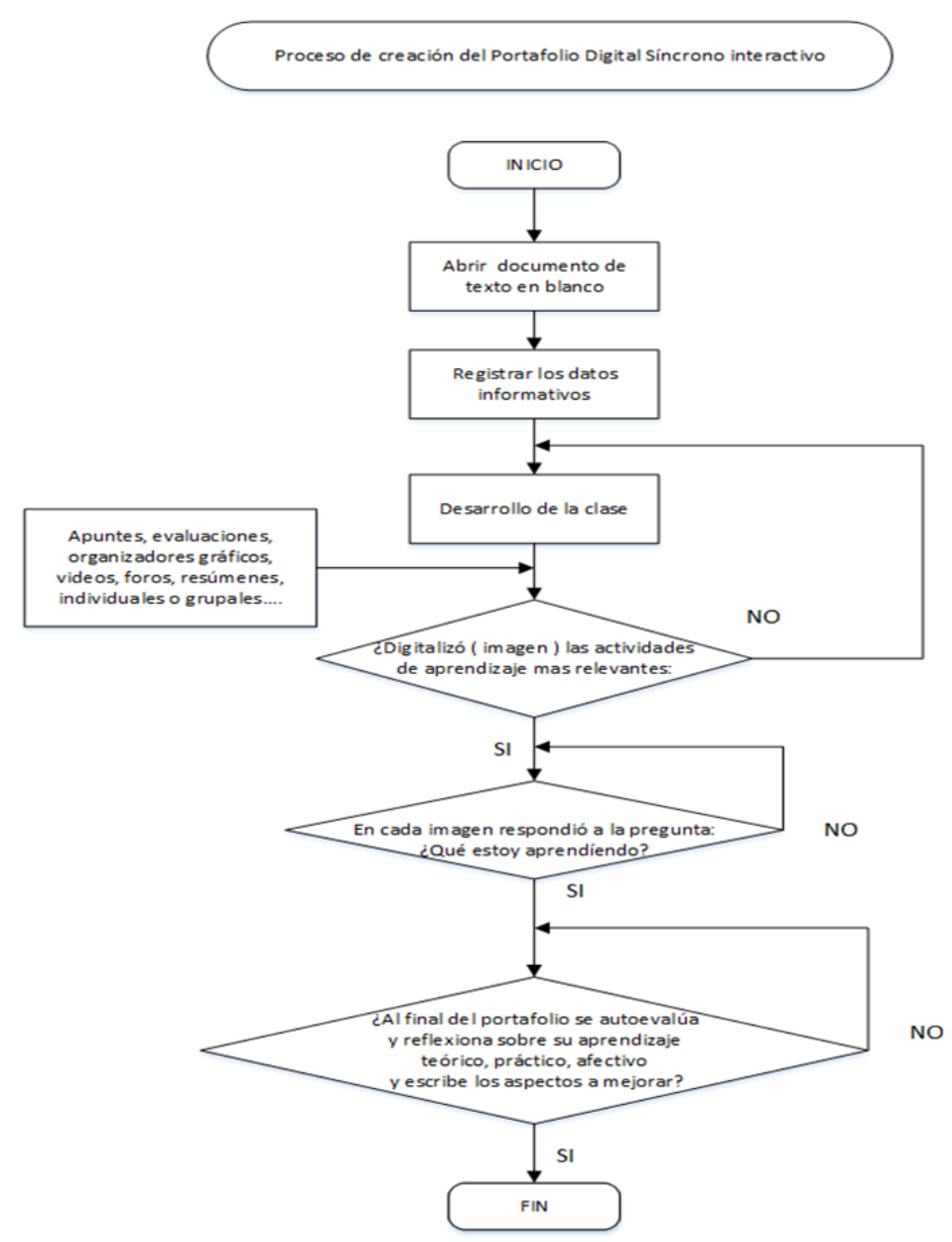

Figura 1. Proceso de creación del Portafolio Digital Interactivo Fuente: Elaboración propia (2021)

En la Figura 1, se observa que el portafolio digital interactivo es una estrategia metodológica de autoevaluación, que permite identificar los aspectos relevantes en la construcción del aprendizaje de los estudiantes, de forma autónoma. Esta estrategia propone la reflexión continua en relación con los aprendizajes adquiridos, que deben registrarse en pocas líneas. En los encuentros síncronos se seleccionan los aprendizajes relevantes, mediante imágenes y se los recopila en un documento de texto, incluyendo una reflexión de lo aprendido, en no más, de dos líneas. Las actividades de aprendizaje son digitalizaciones, que a juicio del estudiante son las más representativas de su aprendizaje, estas digitalizaciones son: apuntes, ejercicios en clase, trabajo en equipo, deberes, evaluaciones, aplicación de simuladores, evidencias del desarrollo de proyectos y demás actividades propias del aprendizaje. Al final del portafolio digital interactivo de cada clase, en pocas líneas, se efectúan reflexiones integrales, relacionadas con los aprendizajes cognitivos, prácticos y valorativos, además de citar los puntos donde el estudiante, según su criterio, debe mejorar. Esta autoevaluación estimula la reflexión autoevaluativa continua del aprendizaje (Tipán, 2021).

Sobre la base de las consideraciones anteriores, es necesario implementar la estrategia metodológica descrita, para desarrollar la cultura autoevaluativa en los estudiantes, aspecto importante, que no ha sido abordado de forma directa hasta la fecha. Por todo lo anterior es necesario incluir el portafolio digital interactivo, en los encuentros síncronos, como herramienta para desarrollar la conciencia autoevaluativa en los estudiantes y futuros profesionales.

\section{Materiales y métodos \\ Participantes}

La población participante en la investigación fue de 70 estudiantes de los tres primeros semestres, de los cuales 59\% son hombres y $41 \%$ mujeres, no se trabajó con una muestra, sino que participaron todos los actores. El $77 \%$ tiene el título de bachillerato general unificado, el $7 \%$ de Bachillerato Internacional, el $13 \%$ Bachillerato técnico y el $3 \%$ provienen de otro bachillerato. Un $29 \%$ de los estudiantes cursa su primer semestre, un $71 \%$ tiene experiencia de uno o más semestres de haber cursado estudios en la actual Universidad o en otras. La edad de los participantes, de forma mayoritaria, oscila entre los 17 y 25 años. (entre 17 y 19: 50\%; entre 20 y 22: 34\%), en las disciplinas de: física. matemática y geometría plana, en la Universidad Tecnológica Indoamérica en las Facultades de Arquitectura y Derecho.

Tabla 1. Población de estudio

\begin{tabular}{cccccc}
\hline $\begin{array}{c}\text { Población por } \\
\text { género }\end{array}$ & $\begin{array}{c}\text { Bachillerato } \\
\text { General } \\
\text { Unificado }\end{array}$ & $\begin{array}{c}\text { Bachillerato } \\
\text { Internacional }\end{array}$ & $\begin{array}{c}\text { Bachillerato } \\
\text { Técnico }\end{array}$ & Otro & $\begin{array}{c}\text { Total } \\
\text { general }\end{array}$ \\
\hline $\begin{array}{c}\text { Femenino } \\
\text { Masculino }\end{array}$ & $37 \%$ & $1 \%$ & $3 \%$ & $0 \%$ & $41 \%$ \\
\hline Total general & $\mathbf{7 7 \%}$ & $6 \%$ & $10 \%$ & $3 \%$ & $59 \%$ \\
\hline \multicolumn{7}{c}{ Fuente: Elaboración propia $(2021)$} & $\mathbf{3 \%}$ & $\mathbf{1 0 0 \%}$ \\
\hline
\end{tabular}




\section{Instrumento}

Los instrumentos de recolección de datos fueron dos; un cuestionario, compuesto con una escala de Likert de 5 opciones:1: Totalmente en desacuerdo; 2: En desacuerdo; 3 : Ni de acuerdo ni en desacuerdo; 4: De acuerdo y 5: Totalmente de acuerdo. La validación del instrumento se efectuó en un taller, con la participación de un grupo de tres expertos en el área educativa y pedagógica, todos los expertos son docentes investigadores de Universidad, en el área de pedagogía, lenguaje y comunicación e informática educativa; los expertos sugirieron cambios al instrumento, los mismos que se incluyeron antes de la aplicación de la encuesta en la población objetivo.

El otro instrumento fue una rúbrica que evalúa el portafolio digital interactivo; la validación de esta rúbrica fue efectuada por los expertos docentes investigadores universitarios, antes señalados; las observaciones permitieron la mejora de la rúbrica, la cual consideró la taxonomía socioformativa y estuvo compuesta por 5 niveles: preformal, receptivo, resolutivo, autónomo y estratégico. Para la confiabilidad o consistencia interna del instrumento, se aplicó el alfa de Cronbach con el programa SPSS ${ }^{\oplus}$, (Tabla $2)$.

Tabla 2. Coeficiente de Cronbach

\begin{tabular}{cc}
\hline Alfa de Cronbach & $\mathbf{N}$ de elementos \\
\hline, 810 & 10 \\
\hline
\end{tabular}

Fuente: Elaboración propia (2021)

Con relación a la rúbrica que permitió evaluar el portafolio digital interactivo que se sustenta en la taxonomía socioformativa, se muestra cómo fue validada por el grupo de expertos docentes investigadores universitarios (Tabla 2).
Tabla 3. Rúbrica con indicadores y descriptores socioformativos.

\begin{tabular}{|c|c|c|c|c|c|}
\hline & Preformal & Receptivo & Resolutivo & Autónomo & Estratégico \\
\hline & $\begin{array}{l}\text { El estudiante } \\
\text { se acerca a la } \\
\text { resolución del } \\
\text { problema } \\
\text { planteado }\end{array}$ & $\begin{array}{l}\text { Muestra un } \\
\text { abordaje y } \\
\text { registro a los } \\
\text { problemas de } \\
\text { forma mecánica. }\end{array}$ & $\begin{array}{l}\text { Logra resolver } \\
\text { los problemas } \\
\text { sencillos }\end{array}$ & $\begin{array}{l}\text { Los problemas son } \\
\text { expuestos mediante } \\
\text { argumentos } \\
\text { propios, los } \\
\text { resuelve y busca } \\
\text { mejoras }\end{array}$ & $\begin{array}{l}\text { Al resolver los } \\
\text { problemas los hace de } \\
\text { forma creativa e } \\
\text { innovadora de forma } \\
\text { flexible articulando los } \\
\text { saberes y los valores }\end{array}$ \\
\hline Indicadores & 1 & 2 & 3 & 4 & 5 \\
\hline $\begin{array}{l}\text { El contenido } \\
\text { del } \\
\text { portafolio } \\
\text { digital }\end{array}$ & $\begin{array}{l}\text { El contenido } \\
\text { del portafolio } \\
\text { digital, } \\
\text { muestra el } \\
\text { acercamiento } \\
\text { a la resolución } \\
\text { del problema } \\
\text { planteado }\end{array}$ & $\begin{array}{l}\text { El portafolio } \\
\text { describe los } \\
\text { contenidos } \\
\text { desarrollados } \\
\text { durante la clase } \\
\text { por medio de los } \\
\text { retos y los } \\
\text { problemas del } \\
\text { entorno en el } \\
\text { marco de los } \\
\text { proyectos } \\
\text { formativos de } \\
\text { forma mecánica }\end{array}$ & $\begin{array}{l}\text { El contenido del } \\
\text { portafolio, } \\
\text { muestra la } \\
\text { resolución } \\
\text { problemas } \\
\text { relacionados } \\
\text { con los } \\
\text { contenidos del } \\
\text { proyecto } \\
\text { formativo, } \\
\text { mediante los } \\
\text { proyectos con } \\
\text { sus partes } \\
\text { constituyentes } \\
\text { de retos y } \\
\text { problemas } \\
\text { sencillos, son } \\
\text { resueltos. }\end{array}$ & $\begin{array}{l}\text { El portafolio } \\
\text { contiene material } \\
\text { desarrollado } \\
\text { durante las clases } \\
\text { videoconferencia, a } \\
\text { través de la } \\
\text { argumentación de } \\
\text { los proyectos y la } \\
\text { ecología de saberes. }\end{array}$ & $\begin{array}{l}\text { Innova mediante la } \\
\text { presentación de los } \\
\text { contenidos, que se } \\
\text { muestran en el portafolio } \\
\text { por medio de los retos y } \\
\text { los problemas del } \\
\text { entorno, como parte de } \\
\text { los proyectos en el } \\
\text { ámbito de la Ecología de } \\
\text { saberes, la creatividad y } \\
\text { los valores. }\end{array}$ \\
\hline $\begin{array}{l}\text { Aplicación } \\
\text { de las TIC y } \\
\text { originalidad } \\
\text { en el } \\
\text { portafolio } \\
\text { digital }\end{array}$ & $\begin{array}{l}\text { Rotula las } \\
\text { imágenes del } \\
\text { portafolio } \\
\text { digital se } \\
\text { aproxima a } \\
\text { resolver lo } \\
\text { planteado en } \\
\text { el problema } \\
\text { mediante las } \\
\text { TIC }\end{array}$ & $\begin{array}{l}\text { Reproduce la } \\
\text { imagen de } \\
\text { forma original } \\
\text { desde un } \\
\text { abordaje } \\
\text { mecánico y con } \\
\text { la utilización de } \\
\text { las Tic }\end{array}$ & $\begin{array}{l}\text { Aplica diversas } \\
\text { herramientas } \\
\text { para la creación } \\
\text { de su portafolio } \\
\text { utilizando las } \\
\text { TIC logrando } \\
\text { resolver } \\
\text { problemas } \\
\text { sencillos }\end{array}$ & $\begin{array}{l}\text { Ejemplifica de } \\
\text { forma original los } \\
\text { diversos momentos } \\
\text { de aprendizaje } \\
\text { frente a los } \\
\text { problemas } \\
\text { propuestos con } \\
\text { argumentos y } \\
\text { mejoras, aplicando } \\
\text { las TIC }\end{array}$ & $\begin{array}{l}\text { Propone de forma } \\
\text { original su portafolio } \\
\text { utilizando diversas } \\
\text { herramientas y } \\
\text { estrategias mediante las } \\
\text { TIC, articulando con los } \\
\text { valores universales para } \\
\text { resolver problemas de } \\
\text { forma creativa e } \\
\text { innovadora. }\end{array}$ \\
\hline $\begin{array}{l}\text { Reflexionar } \\
\text { sobre la } \\
\text { autoevaluaci } \\
\text { ón y mejora } \\
\text { del } \\
\text { aprendizaje } \\
\text { con el } \\
\text { portafolio } \\
\text { digital }\end{array}$ & $\begin{array}{l}\text { Señala sus } \\
\text { reflexiones } \\
\text { i sobre lo } \\
\text { aprendido en } \\
\text { la clase, está } \\
\text { cercana la } \\
\text { resolución a } \\
\text { los ejercicios y } \\
\text { problemas } \\
\text { propuestos }\end{array}$ & $\begin{array}{l}\text { Describe cómo } \\
\text { desarrolló su } \\
\text { aprendizaje, se } \\
\text { autoevalúa y } \\
\text { señala aspectos } \\
\text { de mejora a su } \\
\text { aprendizaje, } \\
\text { desde una óptica } \\
\text { de resolución de } \\
\text { problemas } \\
\text { mecánicamente }\end{array}$ & $\begin{array}{l}\text { Implementa } \\
\text { reflexiones y } \\
\text { temas de mejora } \\
\text { a su aprendizaje, } \\
\text { en la creación } \\
\text { del portafolio } \\
\text { digital; logrando } \\
\text { resolver } \\
\text { sencillos } \\
\text { problemas en el } \\
\text { contexto de la } \\
\text { autoevaluación }\end{array}$ & $\begin{array}{l}\text { Aporta con } \\
\text { reflexiones y } \\
\text { componentes de } \\
\text { mejora a su } \\
\text { aprendizaje, que } \\
\text { constan en su } \\
\text { portafolio para la } \\
\text { resolución de } \\
\text { problemas, } \\
\text { aplicando rúbricas y } \\
\text { argumentando sus } \\
\text { respuestas en busca } \\
\text { de una mejora }\end{array}$ & $\begin{array}{l}\text { Crea reflexiones } \\
\text { profundas respecto de su } \\
\text { aprendizaje y mejora, } \\
\text { resolviendo problemas } \\
\text { con creatividad en el } \\
\text { contexto de la Auto y } \\
\text { Coevaluación utilizando } \\
\text { rúbricas. }\end{array}$ \\
\hline
\end{tabular}

Fuente: Elaboración propia (2021)

\section{Tipo y Diseño}

En una investigación de tipo aplicado, se propone la resolución de la problemática de la autoevaluación de los estudiantes. El diseño de la investigación, tiene como objetivo, proponer una estrategia que permita recabar los datos y la información, para responder a la pregunta de investigación (Hernández-Sampieri y Torres, 2018).

\section{Procedimiento}

El procedimiento de la investigación, se describe en la figura 3. 


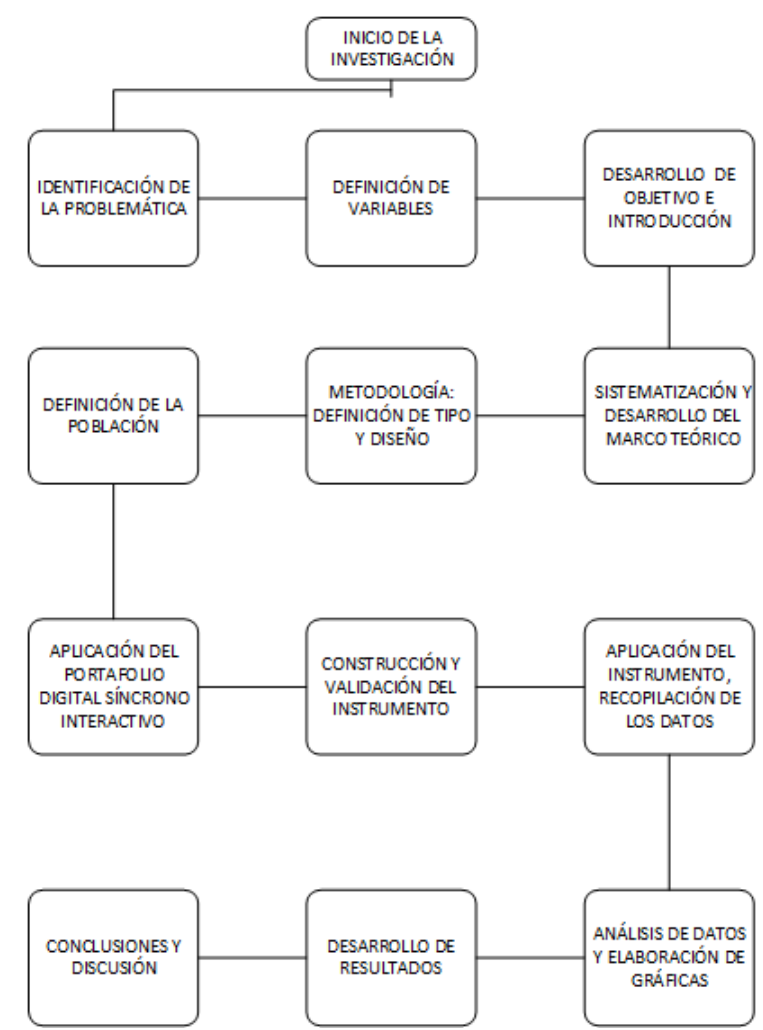

Figura 2. Proceso de investigación.

Fuente: Elaboración propia (2021)

\section{Confidencialidad o Consentimiento informado}

La participación de los estudiantes se realizó, durante el curso de ciencias básicas, en las Facultades de Arquitectura y Derecho, al ser un proceso inédito, los estudiantes dieron su consentimiento para la investigación, habiéndose informado de la realización del proceso investigativo, no se requirió ningún dato, más allá de los propios de la investigación y la experiencia de la aplicación del portafolio digital.

\section{Resultados}

Luego de la implementación del portafolio digital interactivo como estrategia metodológica para la autoevaluación de los aprendizajes y de la aplicación de la encuesta se obtuvieron los siguientes resultados en tres dimensiones de autoevaluación, redefinida mediante la aplicación del portafolio digital, la primera: autoevaluación integral, la segunda: enseñanza aprendizaje y autoevaluación; y la tercera dimensión: el portafolio digital.

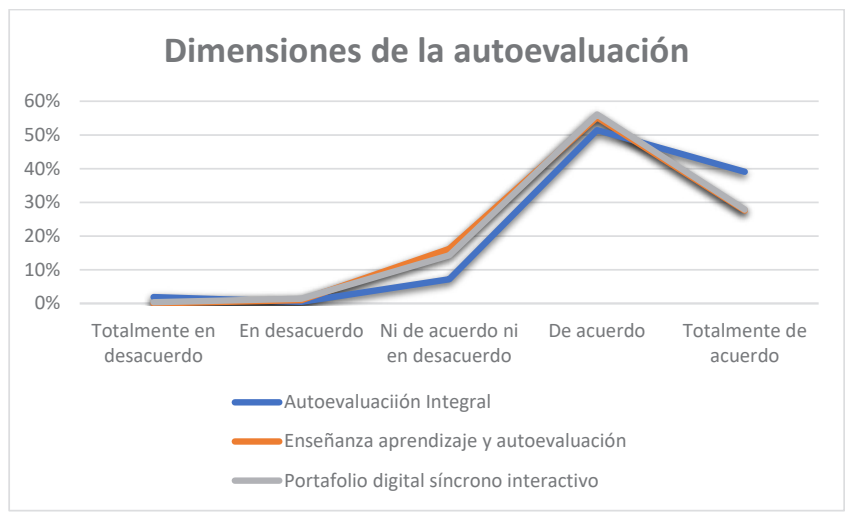

Figura 3. Dimensiones de la Autoevaluación desde la aplicación del portafolio digital

Fuente: Elaboración propia (2021)

Los resultados obtenidos en la Figura 3 muestran que en el caso del primer dominio el $90 \%$ de los participantes está de acuerdo que el portafolio permite la autoevaluación integral, al autoevaluar los dominios cognitivos, afectivos y prácticos. En la segunda dimensión el 83\% está de acuerdo con la necesidad de promover los procesos de autoevaluación dentro del aprendizaje y la planificación docente. En la tercera dimensión, de la autoevaluación del aprendizaje y el portafolio digital interactivo, el $84 \%$ está de acuerdo con el registro de las reflexiones de lo aprendido en las clases virtuales y con la implementación del portafolio en las clases virtuales.

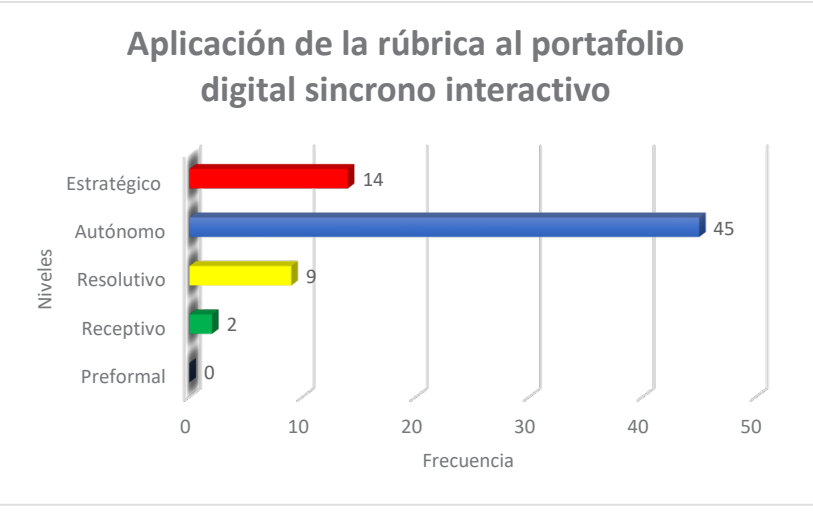

Figura 4. Resultados de la aplicación de la rúbrica Fuente: Elaboración propia (2021)

De la Figura 4, se puede analizar la relación que existe entre número de estudiantes y la autoevaluación en relación con la aplicación del portafolio digital; 14 (20\%) estudiantes consideran que están en el nivel estratégico, 
45 (64\%) estudiantes en el nivel autónomo, 9 (13\%) estudiantes en el nivel resolutivo y 2 (3\%) estudiantes en el nivel receptivo. De lo anterior se interpreta que los estudiantes, como consecuencia de la aplicación del portafolio digital síncrono interactivo, con la rúbrica respectiva, han desarrollado su conciencia autoevaluativa.

\section{Conclusiones}

El logro de desarrollar la reflexión e identificar las mejoras para el aprendizaje síncrono, se alcanza gracias a la implementación del portafolio digital interactivo, como se corrobora en los resultados obtenidos, al analizar las dimensiones de la autoevaluación integral, enseñanza aprendizajeautoevaluación y portafolio digital síncrono; se obtienen porcentajes de acuerdo con valores del $90 \%, 83 \%$ y $85 \%$, respectivamente.

La aplicación de la autoevaluación mediante la rúbrica socioformativa, produjo resultados de un porcentaje mayoritario: 97\% (68) de los estudiantes se consideran estar ubicados en los niveles resolutivo, autónomo y estratégico y muestran su acuerdo y madurez autoevaluativa al reflexionar sobre sus propios portafolios, como consecuencia de su trabajo en los encuentros de las videoconferencias.

Lo datos indican que, a diferencia de otras investigaciones, es posible implementar la herramienta en la virtualidad; queda por verificar si en la presencialidad post-Covid el portafolio digital síncrono interactivo, se puede aplicar para obtener los resultados antes descritos, con las adaptaciones del caso o si no es posible su implementación.

Los resultados señalan que, a diferencia de la perspectiva tradicional, el portafolio deja de ser un repositorio de pruebas, y se convierte en una herramienta dinámica y pertinente para el desarrollo de la autoevaluación; queda planteada la inquietud de si es posible la extrapolación de la estrategia hacia otras disciplinas y público, en los diferentes niveles educativos.

El portafolio digital interactivo y la autoevaluación, han tenido la condición generalizada de repositorios de evidencias, del trabajo desarrollado durante un tiempo y disciplina determinada. Desde la perspectiva del sustento teórico se constató que no existe un recurso como el portafolio digital interactivo, aplicado de la forma como se lo efectuó en la presente investigación, convirtiéndose en un instrumento válido por los resultados obtenidos, que permite redefinir y fortalecer el campo de la autoevaluación tan poco trabajado, y sobre el cual no se han desarrollado investigaciones a profundidad, ni tampoco se han propuesto recursos o estrategias que permitan, en la virtualidad, autoevaluar, más allá de los bancos de preguntas y reflexiones aisladas e inconexas o parciales, que han sido el común denominador de la autoevaluación en la virtualidad.

La presencia de la pandemia, y la inmersión en el proceso enseñanza aprendizaje de forma íntegra en la virtualidad, obligó a que se desarrollen recursos yestrategias metodológicas innovadoras, fruto de esta situación emergió el portafolio digital interactivo, para abordar la problemática de la falta de una cultura autoevaluativa holística en los estudiantes; la herramienta propuesta es el resultado de la nueva realidad de la educación.

La información obtenida luego de incluir el portafolio en las clases virtuales síncronas, permite concluir que se fomentó la conciencia autoevaluativa en los estudiantes. Se logró que reflexionen acerca de sus aprendizajes durante las horas de clases virtuales, mediante el registro de las digitalizaciones de las actividades más representativas, seleccionadas de forma individual, por parte de los estudiantes.

La rúbrica elaborada en una escala de valoración, en el marco de la taxonomía socioformativa, permitió asignar una calificación, a la elaboración del portafolio digital síncrono. La calificación evidenció, el grado de compromiso, auto retroalimentación y reflexión, alrededor de los aprendizajes que los estudiantes alcanzaron; es importante notar que se va desarrollando su madurez, responsabilidad y autocrítica, sobre los aspectos de mejora, que son indispensables en el profesional de la actualidad.

Las organizaciones educativas forman estructuras internas de autoevaluación, con fines 
de acreditación tanto para sus facultades, como para la institución, pero no contemplan procesos de autoevaluación en el núcleo de la educación: el aula de clase y el aprendizaje. El portafolio digital interactivo, puede integrarse dentro de los espacios de autoevaluación, como un recurso innovador válido, para generar reflexiones $\mathrm{y}$ mejoras, sobre lo más importante para que los estudiantes aprendan integralmente.

Como sugerencia a futuro se recomienda el implementar el portafolio digital interactivo, no solo en la virtualidad, sino también en lo semipresencial y presencial; en todas las disciplinas. El instrumento se deberá adaptar y contextualizar, para lograr los resultados esperados.

\section{Referencias Bibliográficas}

Abal, A. A., González, A. M., Pérez, P. S., Procopio Rodríguez, M. M., \& Tanevitch, A. M. (2020). La calificación y la autoevaluación. In IV Jornadas de Actualización en Prácticas Odontológicas Integradas PPS-SEPOI (La Plata, 7 de julio de 2020). http://sedici.unlp.edu.ar/handle/10915/109031

Aguiar, P., B. O. y Velázquez, Á., R. M. (2018). Aproximación teórica al estudio de las tecnologías y su importancia en el proceso de evaluación universitaria. Revista Cubana de Educación Superior, 37(3), e8. http://scielo.sld.cu/pdf/rces/ v37n3/0257-4314-rces-37-03-e8.pdf

Alonso, M. I. G., Domínguez-Fernández, R., Diez-Fernández, Á., Pérez-Pueyo, Á., \& Gutiérrez-García, C. (2019). Autoevaluación y coevaluación de la práctica docente del profesorado universitario. Revista Infancia, Educación y Aprendizaje, 5(2), 353-358.

https://doi.org/10.22370/ieya.2019.5.2.1724

Amaro D. C., R., y Chacín A., R. (2017). La evaluación en el aula virtual. Voces de la educación, 2(1), 3-30. https://hal.archives-ouvertes.fr/hal02540342/document

Amorós-Poveda, L. (2020). Competencia digital docente en Prácticum desde la autoevaluación. Revista Prácticum, 5(2), 30-46. https://revistas. uma.es/index.php/iop/article/view/10235
Archuby, F. H., Sanz, C. V., y Pesado, P. M. (2017). Desafíate: juego serio para la autoevaluación de los alumnos y su integración con un entorno virtual de enseñanza y aprendizaje. In Congreso Argentino de Ciencias de la Computación, 23, 285-294. https://digital.cic.gba.gob.ar/bitstream/ handle/11746/9019/Documento_completo. pdf-PDFA.pdf? sequence $=1$

Ayzum, E., J. M. (2017). La autoevaluación docente de aula: un camino para mejorar la práctica educativa. Revista Electrónica Diálogos Educativos, 11(22), 183-196. http://dialogoseducativos. umce.cl/articulos/2011/dialogos-e-22-aysum. pdf

Calle-Álvarez, G. Y. (2020). La rúbrica de autoevaluación como estrategia didáctica de revisión de la escritura. Revista de Investigación Desarrollo e Innovación: RIDI, 10(2), 323-335. https:// doi.org/10.19053/20278306.v10.n2.2020.10628 Carlos, G., J. J., Martínez V., M. F., y Verdejo M., M. E. (2017). Efectos de enseñar activamente contenidos psicológicos y autoevaluación estudiantil. En XIX Congreso de Investigación Educativa.

Casas, M. J. M. (2020). Estrategia de autoevaluación para mejorar la calidad del desempeño docente en el colegio Militar Elias Aguirre (Tesis de maestría). Universidad Señor de Sipán, Pimentel, Perú. http://repositorio.uss.edu.pe/handle/20.500.12802/6881

Ceballo, R. W. O. (2018). Recursos de autoevaluación sobre amplificadores para el curso virtual de la asignatura Electrónica Analógica I (Tesis de pregrado). Universidad Central" Marta Abreu" de Las Villas, Santa Clara, Cuba. https://dspace. uclv.edu.cu/handle/123456789/10031

Coria, A. I. (2017). Propuesta de un instrumento para la autoevaluación bioética de proyectos de investigación en medicina. Archivos en Artículo Original, 19(4), 121-131. https://web.archive. org/web/20180518174500id_/http://www.medigraphic.com/pdfs/medfam/amf-2017/amf174g. pdf

Dooner, C., Armanet, L., Busco, C., d'Alencon, A. y Salomone, A. (2016). Impacto de los procesos de autoevaluación en la gestión de pregrado y postgrado en la Universidad de Chile (2011- 
2014). Santiago de Chile: Comisión Nacional de Acreditación. Serie Cuadernos de Investigación en Aseguramiento de la Calidad, 216.

Fernández-Jiménez, C., Polo, M. y Fernández Cabezas, M. (2017). Aplicación de la autoevaluación en una experiencia de Aprendizaje Basado en Problemas con alumnado de educación en asignaturas relacionadas con la discapacidad. Estudios sobre educación, 32, 73-93. https://doi. org/10.15581/004.32.73-93

Fosado, Q., R. E., Martínez R., A., Hernández N., N., y Ávila R., R. (2018). El portafolio virtual como una herramienta transversal de planeación y evaluación del aprendizaje autónomo para el desarrollo sustentable. RIDE. Revista Iberoamericana para la Investigación y el Desarrollo Educativo, 8(16), 194-215. http://www.scielo.org.mx/ scielo.php?pid=S2007-74672018000100194\&script=sci_arttext

García-Beltrán, Á., Martínez, R., Jaén, J. A. y Tapia, S. (2016). La autoevaluación como actividad docente en entornos virtuales de aprendizaje/ enseñanza. Revista de Educación a Distancia (RED), (50).

https://doi.org/10.6018/red/50/14

García-Mauriño, M., J. E. (2017). Prácticas de Histología Humana: Microscopio Virtual y Autoevaluación. Universidad Complutense de Madrid. https://eprints.ucm.es/id/eprint/43646/

Hernández-Sampieri, R., y Torres, C. P. M. (2018). Metodología de la investigación (Vol. 4). México: McGraw-Hill Interamericana.

https://doi.org/10.17993/CcyLl.2018.15

Herrera, L. S., Hernández, M. G., Pérez, B. M. H., Camiño, R. R., y Lorenzo, A. R. (2020). El portafolio electrónico como herramienta para el aprendizaje en red. Panorama Cuba y Salud, 15(2), 39-44. https://www.medigraphic.com/cgi$\mathrm{bin} /$ new/resumen.cgi?IDARTICULO=99624

Howlet, L. C. P., Aguilar, A. L. S., y Diez, M. D. C. G. (2019). Autoevaluación de docentes en competencias tecno-pedagógicas para la elaboración de materiales didácticos virtuales. Publicaciones: Facultad de Educación y Humanidades del Campus de Melilla, 49(5), 161-177. https:/doi. org/10.30827/publicaciones.v49i5.8318
Kambourova, M. (2018). Una mirada hacia la autoevaluación del aprendizaje en la educación superior. CIAIQ2018, 1, 160-165. https://proceedings.ciaiq.org/index.php/ciaiq2018/article/ view/1637/1590

Kambourova, M. (2020). ¿Qué falta por comprender sobre el concepto autoevaluación (del aprendizaje) en educación superior? Una mirada diferente desde su historia. Avaliação: Revista da Avaliação da Educação Superior (Campinas), 25(3), 640-658.

ht tps://doi.org/10.1590/s 1414 40772020000300007

López-de-Arana P., E., Aramburuzabala H., P. y Opazo C., H. (2020). Diseño y validación de un cuestionario para la autoevaluación de experiencias de aprendizaje-servicio universitario. Educación XX1, 23(1), 319-347.

https://doi.org/10.5944/educxx1.23834

Marciniak, R. y Sallán, J. G. (2017). Un modelo para la autoevaluación de la calidad de programas de educación universitaria virtual. Revista de Educación a Distancia (RED), (54). https:// doi.org/10.6018/red/54/2

Márquez, A. M. B. (2017). La evaluación de la educación a distancia: propuesta de una guía para la autoevaluación. HAMUT’AY, 4(1), 31-44. https://doi.org/10.21503/hamu.v4i1.1394

Martínez-Izaguirre, M., de Eulate, C. Y. Á. y Villardón-Galleg, L. (2018). Autoevaluación y reflexión docente para la mejora de la competencia profesional del profesorado en la sociedad del conocimiento. Revista de Educación a Distancia (RED), (56). https://doi.org/10.6018/red/56/10

Mendoza, S. T. B., Cedeño, J. A. M., Espinales, A. N. V. y Gámez, M. R. (2021). Autoevaluación, Coevaluación y Heteroevaluación como enfoque innovador en la práctica pedagógica y su efecto en el proceso de enseñanza-aprendizaje. Polo del Conocimiento: Revista científico-profesional, 6(3), 828-845. https://dialnet.unirioja.es/servlet/ articulo? codigo $=7926891$

Montaño, F. D. (2021). Evaluación de herramientas digitales para la gestión del portafolio educativo. Minerva, 2(4), 55-61.

https://doi.org/10.47460/minerva.v2i4.27

Padilla, M. Y. M. (2017). La autoevaluación como 
estrategia de aprendizaje en el entorno virtual del curso Derecho Civil IV en la Universidad Abierta para Adultos. Educación Superior, 16(23), 105121. http://rai.uapa.edu.do:8080/xmlui/handle/123456789/358

Rico-Reintsch, K. I. (2019). Uso de autoevaluación docente como herramienta innovadora para el mejoramiento de las asignaturas universitarias (Using Faculty Self-Evaluation As An Innovative Tool to Improve University Courses). Revista CEA, 5(10). https://doi.org/10.22430/24223182.1445

Rodríguez, R. G. (2019). Apuntes sobre la evaluación, la neurociencia y la autoevaluación en la formación de docentes de la universidad nacional de educación. Revista Electrónica Entrevista Académica (REEA), 1(4), 158-167. https://dialnet. unirioja.es/servlet/articulo? codigo $=7808865$

Ruano, M. D. C. B. y Ballesteros, E. P. (2017). El portafolio virtual y su relevancia en trabajos colaborativos. Revista Docencia Universitaria, 18(2), 53-68. https://revistas.uis.edu.co/index. $\mathrm{php} /$ revistadocencia/article/view/9308

Salom, M. A. C. (2018). La autoevaluación. Una propuesta formativa e innovadora. Revista Iberoamericana de Educación, 76(2), 135-152.

https://doi.org/10.35362/rie7623081

Sierra, R. A. C. (2020). La autoevaluación para el empoderamiento de los estudiantes de educación superior virtual. Universidad Militar Nueva Granada. https://repository.unimilitar.edu.co/handle/10654/37855

Tipán, D. (2021). El portafolio digital síncrono interactivo. Revista Andina De Educación, 4(1), 83-89. https://doi.org/10.32719/26312816.2020. 4.1 .10

Vera, J. Á., Bueno C., G., Calderón, N. G. y Medina, F. L. (2018). Modelo de autoevaluación y heteroevaluación de la práctica docente en Escuelas Normales. Educação e Pesquisa, 44.

Vera, A. D. P., Castiblanco J., I. A. y Cruz G., J. P. (2018). Desarrollo de Modelo integral de Autoevaluación en una Institución de Educación Superior. Praxis \& Saber, 9(21), 221-245. https://doi. org/10.19053/22160159.v9.n21.2018.8047

Villarroel, K. y Hernández M., T. (2019). Fundamentos históricos de procesos de autoeva- luación y certificación en Bolivia. Fides et Ratio-Revista de Difusión cultural y científica de la Universidad La Salle en Bolivia, 17(17), 165192. http://www.scielo.org.bo/scielo.php?pi$\mathrm{d}=$ S2071-081X2019000100009\&script=sci_abstract\&tlng=en 